\title{
An efficient and simple approach for the synthesis of pyranopyrazoles using imidazole (catalytic) in aqueous medium, and the vibrational spectroscopic studies on 6-amino-4-(4'-methoxyphenyl)-5-cyano-3-methyl-1-phenyl-1,4- dihydropyrano[2,3-c]pyrazole using density functional theory
}

\author{
Aisha Siddekha ${ }^{\mathrm{b}}$, Aatika Nizam ${ }^{\mathrm{a}}$, M.A. Pasha ${ }^{\mathrm{a}, *}$ \\ a Department of Studies in Chemistry, Central College Campus, Bangalore University, Bangalore 560001, India \\ ${ }^{\mathrm{b}}$ Department of Chemistry, Smt. V.H.D. Central Institute of Home Science, Bangalore 560001, India
}

\section{A R T I C L E I N F O}

\section{Article history:}

Received 23 November 2010

Received in revised form 10 June 2011

Accepted 16 June 2011

\section{Keywords:}

Pyranopyrazoles

Imidazole

Aldehydes

Hydrazine hydrate

Ethyl acetoacetate

Vibrational spectroscopic analysis

\begin{abstract}
A B S T R A C T
We describe a one-pot four component synthesis of pyranopyroles from aryl aldehydes, ethyl acetoacetate, malononitrile and hydrazine hydrate in the presence of catalytic amounts of an organocatalyst imidazole in water as medium. A plausible mechanism for the formation of imidazole catalyzed pyranopyrazoles has been envisaged. This method is rapid, simple, provides products in good yield, and is eco-friendly. In addition, based on the optimized geometry, the frequency and intensity of the vibrational bands of 6-amino-4-(4'-methoxyphenyl)-5-cyano-3-methyl-1-phenyl-1,4dihydropyrano[2,3-c]pyrazole were obtained by the density functional theory (DFT) calculations using $6-31 G(d, p)$ basis set. The vibrational frequencies were calculated and the scaled values have been compared with experimental FT-IR and FT-Raman spectra. The observed and the calculated frequencies are found to be in good agreement.
\end{abstract}

(c) 2011 Elsevier B.V. All rights reserved.

\section{Introduction}

Pyranopyrazoles are fused heterocyclic compounds that possess many biological properties such as fungicidal [1], bactericidal [2], vasodilatory activities [3] and act as anticancer agents [4]. They also find application as pharmaceutical ingredients and biodegradable agrochemicals [5-8]. Apart from this, pyrano[2,3-c]pyrazoles have been shown to act as potential insecticidal [9a] and molluscicidal agents [9b]. As a result, considerable attention has been focused on the development of new methodologies for the synthesis of these heterocycles.

Multi-component reactions (MCRs), are those reactions in which three or more reactants react together to give the product in a single step under suitable reaction conditions [10]. MCRs offer the advantage of simplicity and synthetic efficiency over conventional chemical reactions. The MCRs have the additional advantages of selectivity, synthetic convergency, and atom-economy [11a,b]. Further, use of water as a solvent is an active area of research in

\footnotetext{
* Corresponding author. Tel.: +91 8022961337.

E-mail addresses: mafpasha@gmail.com, m_af_pasha@ymail.com (M.A. Pasha).
}

green chemistry. A number of classic reactions which were carried out strictly under anhydrous conditions, in hazardous and toxic organic solvents, can also be carried out in water with proper use of catalysts and reaction conditions. MCRs which are carried out in water as a medium offer better environmental protection, hence, are considered as clean and green reactions.

Pyranopyrazole was first synthesized by the reaction between 3-methyl-1-phenylpyrazolin-5-one and tetracyanoethylene [5]. Sharanin et al. later reported a one-pot three component reaction between pyrazolone, aromatic aldehyde and malononitrile in the presence of triethyl amine as a catalyst in ethanol to get pyranopyrazoles [7]. Another method has been reported by the condensation of $\mathrm{N}$-methylpiperidone, pyrazoline-5-one and malononitrile in absolute ethanol [12]. Nadia et al. reported a method using a mixture of 5-methyl-2,4-dihydro-3H-pyrazol3-one, malononitrile and different aromatic aldehydes in the presence of ammonium acetate in ethanol [4]. A four component reaction of aldehydes, ethyl acetoacetate, malononitrile and hydrazine hydrate in water and catalytic $\beta$-cyclodextrin was reported by Vasuki and Kandhasamy [8] and the same reaction is carried out by Shestopalov et al. using catalytic amounts of triethyl amine in ethanol [13]. However, all the reported 


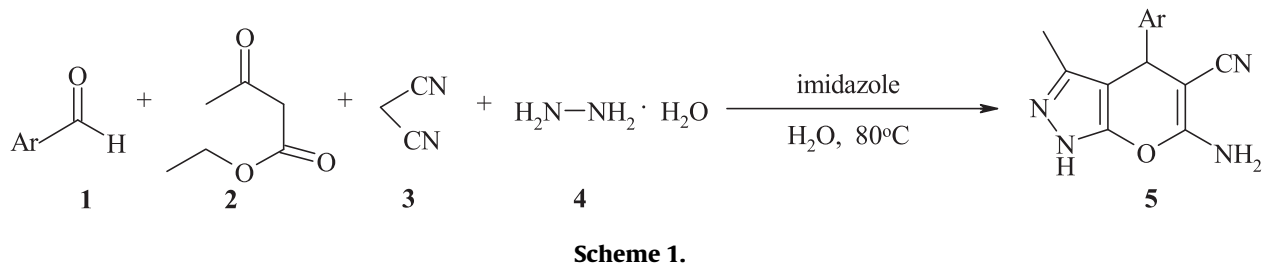

methods are associated with disadvantages such as: use of expensive and environmentally hazardous reagents, low yields of products, drastic reaction conditions and tedious work-up procedures.

In continuation of our work on the synthesis of biologically active heterocyclic organic compounds [14a-e]; herein, we are reporting a simple and efficient synthesis of pyranopyrazoles by a four component reaction of aromatic aldehydes (1), ethyl acetoacetate (2), malononitrile (3) and hydrazine hydrate (4) in the presence of an organocatalyst imidazole in water at $80^{\circ} \mathrm{C}$ (Scheme 1). The method is environmentally friendly as it is carried out in water, involves the use of an inexpensive ecofriendly organocatalyst and gives high yield of the products within $30 \mathrm{~min}$.

\section{Results and discussion}

For a detailed exploration, the reaction between $p$-anisaldehyde and ethyl acetoacetate, malononitrile and hydrazine hydrate in the presence of imidazole was considered for the optimization of amount of catalyst used; and to study the effect of solvents on the rate of the reaction. Initially, when the reaction was carried out in the absence of any catalyst, no product was found even after $30 \mathrm{~min}$. Addition of $0.2 \mathrm{mmol}$ imidazole afforded the product in low yield, an increase in the amount of imidazole to $0.5 \mathrm{mmol}$ gave 6 -amino-4-(4'-methoxyphenyl)-5cyano-3-methyl-1-phenyl-1,4-dihydropyrano[2,3-c]pyrazole (5a) in $88 \%$ yield; further increase in the amount of catalyst did not increase the product yield. Next, in order to investigate the effect of solvents, the above reaction was carried out in conventional organic solvents such as $\mathrm{DCM}, \mathrm{CH}_{3} \mathrm{CN}$, ethanol and in water. Water as a solvent provided the best yields compared to $\mathrm{DCM}, \mathrm{CH}_{3} \mathrm{CN}$ and ethanol.

In order to establish the generality, the catalyst was successfully applied to the reaction of various araldehydes with ethyl acetoacetate, malononitrile and hydrazine hydrate and the results of this study are presented in Table 1. It is clear from this table that, excellent product yields were obtained with aryl and heteroaryl aldehydes. Furthermore, the reaction is compatible in the presence of various functional groups such as $-\mathrm{Cl},-\mathrm{OCH}_{3},-\mathrm{NO}_{2}$ and $-\mathrm{OH}$.<smiles>COc1ccc(C2C(C#N)=C(N)Oc3[nH]nc(C)c32)cc1</smiles>

$5 \mathbf{a}$
Table 1

Synthesis of pyranopyrazoles from various aromatic aldehydes, malononitrile, ethyl acetoacetate and hydrazine hydrate.

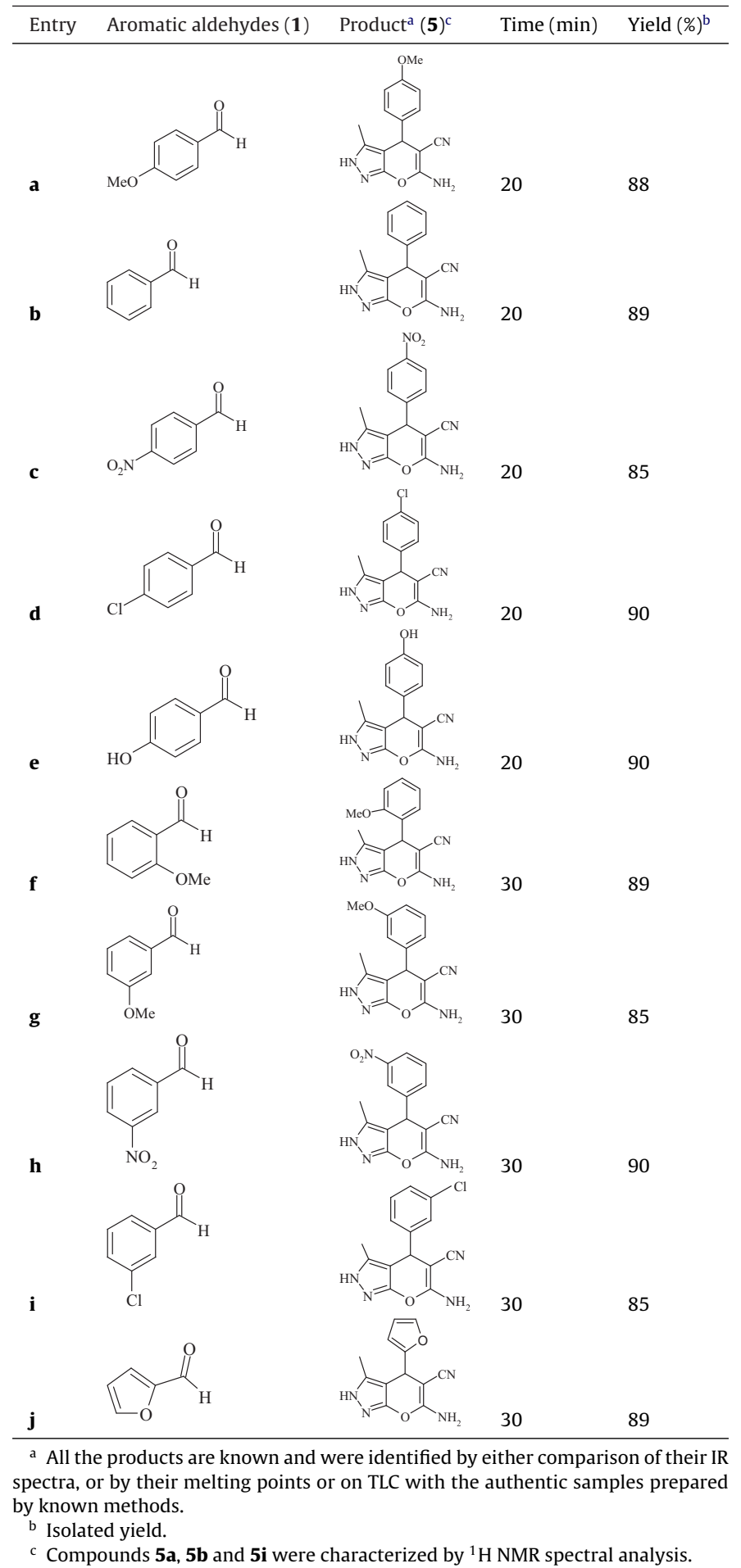

Fig. 1. Structure of 6-amino-4-(4'-methoxyphenyl)-5-cyano-3-methyl-1-phenyl1,4-dihydropyrano[2,3-c]pyrazole $\left(\mathrm{C}_{15} \mathrm{H}_{14} \mathrm{~N}_{4} \mathrm{O}_{2}\right)$. 


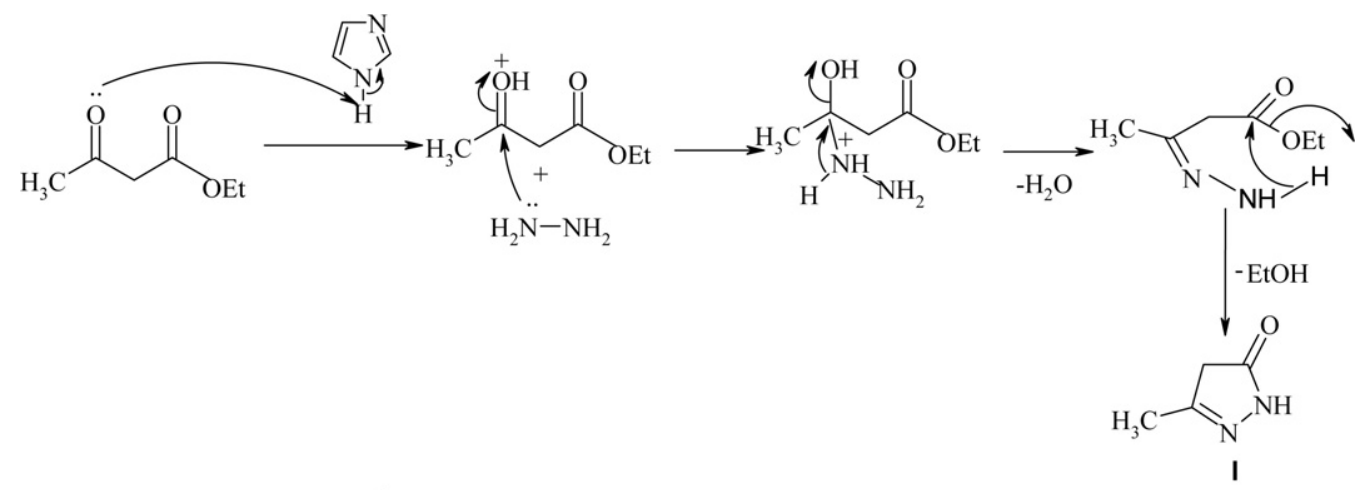

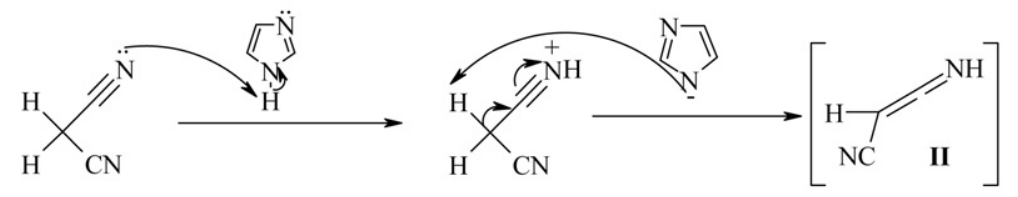

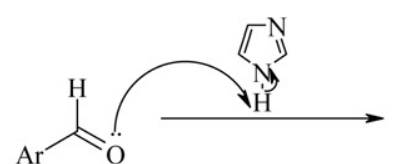

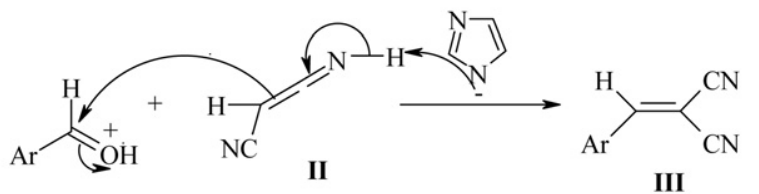<smiles>CC1=NNC(=O)C1</smiles><smiles>CC1=NNC(=O)C1CNCn1ccnc1</smiles><smiles>C1CCCCC1</smiles><smiles>Cc1n[nH]c(O)c1CC(C#N)C#N</smiles><smiles>Cc1n[nH]c2c1C([Al])C(C#N)=C(N)O2</smiles>

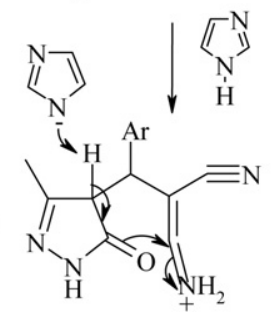

Scheme 2. A plausible mechanism for the formation of pyranopyrazoles.

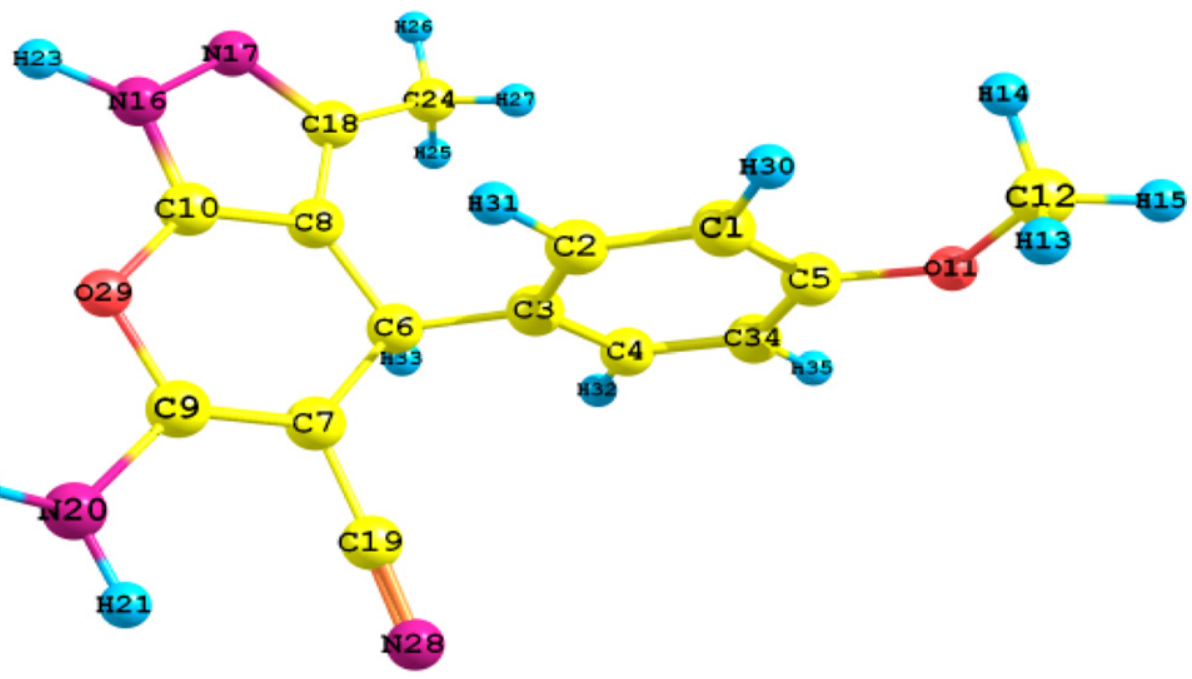

Fig. 2. Numbering system adopted for the optimized structure of 6-amino-4-(4'-methoxyphenyl)-5-cyano-3-methyl-1-phenyl-1,4-dihydropyrano[2,3-c]pyrazole. 
Table 2

Optimized bond lengths and bond angles of 6-amino-4-(4'-methoxyphenyl)-5cyano-3-methyl-1-phenyl-1,4-dihydropyrano[2,3-c]pyrazole.

\begin{tabular}{|c|c|c|c|}
\hline Bond & Length & Angle & $\left({ }^{\circ}\right)$ \\
\hline $\mathrm{C} 1-\mathrm{C} 2$ & 1.396 & $\mathrm{C} 2-\mathrm{C} 1-\mathrm{C} 5$ & 119.6 \\
\hline $\mathrm{C} 1-\mathrm{C} 5$ & 1.401 & $\mathrm{C} 2-\mathrm{C} 1-\mathrm{H} 30$ & 119.4 \\
\hline C1-H30 & 1.083 & $\mathrm{C} 1-\mathrm{C} 2-\mathrm{C} 3$ & 121.6 \\
\hline $\mathrm{C} 2-\mathrm{C} 3$ & 1.397 & $\mathrm{C} 1-\mathrm{C} 2-\mathrm{H} 31$ & 119.0 \\
\hline C2-H31 & 1.087 & C5-C1-H30 & 121.0 \\
\hline C3-C4 & 1.401 & $\mathrm{C} 1-\mathrm{C} 5-\mathrm{O} 11$ & 124.7 \\
\hline C3-C6 & 1.530 & C1-C5-H34 & 119.5 \\
\hline C4-H32 & 1.087 & C3-C2-H31 & 119.5 \\
\hline C4-C34 & 1.390 & $\mathrm{C} 2-\mathrm{C} 3-\mathrm{C} 4$ & 118.0 \\
\hline C5-011 & 1.365 & $\mathrm{C} 2-\mathrm{C} 3-\mathrm{C} 6$ & 121.5 \\
\hline C5-C34 & 1.401 & C4-C3-C6 & 120.5 \\
\hline C6-C7 & 1.539 & С3-C4-H32 & 119.5 \\
\hline $\mathrm{C} 6-\mathrm{C} 8$ & 1.504 & С3-C4-H34 & 121.3 \\
\hline C6-H33 & 1.100 & C3-C6-C7 & 112.0 \\
\hline $\mathrm{C} 7-\mathrm{C} 9$ & 1.368 & C3-C6-C8 & 113.8 \\
\hline C7-C19 & 1.419 & С3-C6-H33 & 106.6 \\
\hline $\mathrm{C} 8-\mathrm{C} 10$ & 1.370 & H32-C4-H34 & 119.2 \\
\hline C8-C18 & 1.426 & C4-H34-C5 & 120.0 \\
\hline C9-N20 & 1.367 & O11-C5-H34 & 115.8 \\
\hline C9-O29 & 1.373 & C5-O11-C12 & 118.3 \\
\hline C10-N16 & 1.349 & C5-H34-H35 & 118.6 \\
\hline C10-029 & 1.363 & C7-C6-C8 & 106.8 \\
\hline $\mathrm{O} 11-\mathrm{C} 12$ & 1.419 & C7-C6-H33 & 107.9 \\
\hline C12-H13 & 1.098 & C6-C7-C9 & 125.1 \\
\hline C12-H14 & 1.098 & C6-C7-C19 & 118.8 \\
\hline C12-H15 & 1.091 & C8-C6-H33 & 109.6 \\
\hline N16-N17 & 1.366 & C6-C8-C10 & 121.9 \\
\hline N16-H23 & 1.007 & C6-C8-C18 & 134.7 \\
\hline N17-C18 & 1.334 & C9-C7-C19 & 116.1 \\
\hline C18-C24 & 1.496 & C7-C9-O20 & 126.3 \\
\hline C19-N28 & 1.167 & C7-C9-O29 & 123.8 \\
\hline N20-H21 & 1.010 & C7-C19-N28 & 177.9 \\
\hline N20-H22 & 1.010 & C10-C8-C18 & 103.4 \\
\hline C24-H25 & 1.096 & C8-C10-N16 & 109.1 \\
\hline C24-H26 & 1.092 & N8-N10-O29 & 128.5 \\
\hline C24-H 27 & 1.095 & N8-C18-N17 & 111.5 \\
\hline \multirow[t]{5}{*}{ C34-H35 } & 1.085 & N8-C18-N24 & 128.1 \\
\hline & & N20-C9-O29 & 109.9 \\
\hline & & C9-N20-H21 & 116.2 \\
\hline & & C9-N20-H22 & 116.0 \\
\hline & & $\mathrm{C} 9-029-\mathrm{C} 10$ & 113.9 \\
\hline
\end{tabular}

\section{Experimental}

All chemicals are commercial and were used without further purification. Progress of the reactions was monitored using Silica gel-H TLC plates. The synthesized compounds were characterized by ${ }^{1} \mathrm{H}$ NMR spectral analysis; by comparing the products on TLC or by the comparison of melting points with products prepared by known methods. NMR spectra were recorded on a Brucker spectrophotometer. FT-IR spectra were recorded on a Bruker Optics Alpha-P FT-IR spectrophotometer with attenuated total reflectance (ATR) module. The FT-Raman were recorded in solid phase on Bruker Optics MultiRAM FT-Raman spectrophotometer using Nd-YAG laser operating at $1064 \mathrm{~nm}$ as an excitation source at the resolution of $4 \mathrm{~cm}^{-1}$. Vibrational spectroscopic studies of 6-amino-4-(4'-methoxyphenyl)-5-cyano-3methyl-1-phenyl-1,4-dihydropyrano[2,3-c]pyrazole (5a) was done by recording FT-IR, FT-Raman spectra in the region $4000-400 \mathrm{~cm}^{-1}$ and $3500-100 \mathrm{~cm}^{-1}$, respectively.

\subsection{Typical procedure for the preparation of pyranopyrazole}

The aromatic aldehyde ( $1 \mathrm{mmol})$, malononitrile ( $1 \mathrm{mmol})$, ethyl acetoacetate $(1 \mathrm{mmol})$ hydrazine hydrate $(1 \mathrm{mmol})$ and imidazole $(0.5 \mathrm{mmol})$ were taken in water $(5 \mathrm{ml})$ and heated on a preheated hot plate at $80^{\circ} \mathrm{C}$ for 20-30 min. Various aromatic aldehydes used,
Table 3

Optimized dihedral angles of 6-amino-4-(4'-methoxyphenyl)-5-cyano-3-methyl-1phenyl-1,4-dihydropyrano[2,3-c]pyrazole.

\begin{tabular}{|c|c|c|c|}
\hline Dihedral angle & $\left({ }^{\circ}\right)$ & Dihedral angle & $\left({ }^{\circ}\right)$ \\
\hline $\mathrm{H} 30-\mathrm{C} 1-\mathrm{C} 2-\mathrm{C} 3$ & 179.8 & C7-C6-C8-C18 & -178.7 \\
\hline $\mathrm{C} 5-\mathrm{C} 1-\mathrm{C} 2-\mathrm{H} 31$ & -179.8 & H33-C6-C8-C10 & 118.2 \\
\hline $\mathrm{H} 30-\mathrm{C} 1-\mathrm{C} 2-\mathrm{H} 31$ & 0.13 & H33-C6-C8-C18 & -62.09 \\
\hline $\mathrm{C} 2-\mathrm{C} 1-\mathrm{C} 5-\mathrm{O} 11$ & 179.8 & $\mathrm{C} 6-\mathrm{C} 7-\mathrm{C} 9-\mathrm{N} 20$ & 176.2 \\
\hline $\mathrm{C} 2-\mathrm{C} 1-\mathrm{C} 5-\mathrm{C} 34$ & 0.058 & C6-C7-C9-O29 & -1.01 \\
\hline H30-C1-C5-O11 & -0.11 & C19-C7-C9-N20 & -3.30 \\
\hline $\mathrm{H} 30-\mathrm{C} 1-\mathrm{C} 5-\mathrm{C} 34$ & -179.9 & C19-C7-C9-O29 & 179.4 \\
\hline $\mathrm{C} 1-\mathrm{C} 2-\mathrm{C} 3-\mathrm{C} 4$ & 0.19 & C6-C8-C10-N16 & 179.75 \\
\hline $\mathrm{C} 1-\mathrm{C} 2-\mathrm{C} 3-\mathrm{C} 6$ & 179.2 & C6-C8-C10-O29 & -1.026 \\
\hline $\mathrm{H} 31-\mathrm{C} 2-\mathrm{C} 3-\mathrm{C} 4$ & 179.9 & C18-C8-C10-N16 & -0.05 \\
\hline $\mathrm{H} 31-\mathrm{C} 2-\mathrm{C} 3-\mathrm{C} 6$ & -1.0841 & $\mathrm{C} 18-\mathrm{C} 8-\mathrm{C} 10-\mathrm{O} 29$ & 179.18 \\
\hline $\mathrm{C} 2-\mathrm{C} 3-\mathrm{C} 4-\mathrm{H} 32$ & 179.7 & C6-C8-C18-N17 & -179.7 \\
\hline $\mathrm{C} 2-\mathrm{C} 3-\mathrm{C} 4-\mathrm{C} 34$ & -0.12 & C6-C8-C18-C24 & 0.26 \\
\hline $\mathrm{C} 6-\mathrm{C} 3-\mathrm{C} 4-\mathrm{H} 32$ & 0.63 & C10-C8-C18-N17 & 0.01 \\
\hline C6-C3-C4-C34 & -179.2 & $\mathrm{C} 10-\mathrm{C} 8-\mathrm{C} 18-\mathrm{C} 24$ & -180 \\
\hline $\mathrm{C} 2-\mathrm{C} 3-\mathrm{C} 6-\mathrm{C} 7$ & -71.72 & C7-C9-N20-H21 & 14.32 \\
\hline $\mathrm{C} 2-\mathrm{C} 3-\mathrm{C} 6-\mathrm{C} 8$ & 49.55 & C7-C9-N20-H22 & 155.5 \\
\hline $\mathrm{C} 2-\mathrm{C} 3-\mathrm{C} 6-\mathrm{H} 33$ & 170.5 & O29-C9-N20-H21 & -168.1 \\
\hline $\mathrm{C} 4-\mathrm{C} 3-\mathrm{C} 6-\mathrm{C} 7$ & 107.3 & O29-C9-N20-H22 & -26.91 \\
\hline $\mathrm{C} 4-\mathrm{C} 3-\mathrm{C} 6-\mathrm{C} 8$ & -131.4 & C7-C9-O29-C10 & 1.682 \\
\hline $\mathrm{C} 4-\mathrm{C} 3-\mathrm{C} 6-\mathrm{H} 33$ & -10.49 & N20-C9-O29-C10 & -176.0 \\
\hline C3-C4-C34-C5 & 0.02 & C8-C10-N16-N17 & 0.07 \\
\hline C3-C4-C34-H35 & 179.9 & C8-C10-N16-H23 & 179.1 \\
\hline H32-C4-C34-C5 & -179.8 & O29-C10-N16-N17 & -179.2 \\
\hline H32-C4-C34-H35 & 0.15 & O29-C10-N16-H23 & -0.23 \\
\hline $\mathrm{C} 1-\mathrm{C} 5-\mathrm{O} 11-\mathrm{C} 12$ & 0.72 & C8-C10-O29-C9 & -0.71 \\
\hline C34-C5-O11-C12 & -179.5 & $\mathrm{~N} 16-\mathrm{C} 10-\mathrm{O} 29-\mathrm{C} 9$ & 178.4 \\
\hline $\mathrm{C} 1-\mathrm{C} 5-\mathrm{C} 34-\mathrm{C} 4$ & 0.01 & $\mathrm{C} 5-\mathrm{O} 11-\mathrm{C} 12-\mathrm{H} 13$ & 60.89 \\
\hline C1-C5-C34-H35 & -180.0 & $\mathrm{C} 5-\mathrm{O} 11-\mathrm{C} 12-\mathrm{H} 14$ & -61.53 \\
\hline O11-C5-C34-C4 & -179.8 & C5-O11-C12-C15 & 179.7 \\
\hline O11-C5-C34-H35 & 0.25 & C10-N16-N17-C18 & -0.06 \\
\hline C3-C6-C7-C9 & 124.6 & H23-N16-N17-C18 & -179.1 \\
\hline C3-C6-C7-C19 & -55.90 & N16-N17-C18-C8 & 0.03 \\
\hline C8-C6-C7-C9 & -0.64 & N16-N17-C18-C24 & -180.0 \\
\hline $\mathrm{C} 8-\mathrm{C} 6-\mathrm{C} 7-\mathrm{C} 19$ & 178.9 & $\mathrm{C} 8-\mathrm{C} 18-\mathrm{C} 24-\mathrm{H} 25$ & 62.10 \\
\hline H33-C6-C7-C9 & -118.4 & $\mathrm{C} 8-\mathrm{C} 18-\mathrm{C} 24-\mathrm{H} 26$ & -177.9 \\
\hline H33-C6-C7-C19 & 61.10 & C8-C18-C24-H27 & -57.39 \\
\hline $\mathrm{C} 3-\mathrm{C} 6-\mathrm{C} 8-\mathrm{C} 10$ & -122.6 & N17-C18-C24-H25 & -117.9 \\
\hline C3-C6-C8-C18 & 57.2 & N17-C18-C24-H26 & 2.06 \\
\hline $\mathrm{C} 7-\mathrm{C} 6-\mathrm{C} 8-\mathrm{C} 10$ & 1.54 & N17-C18-C24-H27 & 122.6 \\
\hline
\end{tabular}

yield of the products and time taken for completion of the reactions is summarized in Table 1.

\subsection{Mechanism}

A plausible mechanism for the formation of imidazole catalyzed pyranopyrazoles is envisaged. The formation of pyranopyrazoles catalyzed by imidazole may involve the protonation of ethyl acetoacetate by imidazole, followed by intermolecular attack by hydrazine hydrate. Subsequent loss of water, and intramolecular nucleophillic attack by $-\mathrm{NH}_{2}$ group on the carbonyl carbon to give 5-methyl-2,4-dihydro-pyrazol-3-one (I). Similarly protonation of aldehyde by imidazole and reaction with 3-imino-acrylonitrile (II) may afford 2-benzylidene-malononitrile (III). Addition of I to III in the presence of imidazole followed by rearrangement may give the expected pyranopyrazole as shown in Scheme 2.

\section{Vibrational spectroscopic studies on 6-amino-4-(4'-methoxyphenyl)-5-cyano-3-methyl-1- phenyl-1,4-dihydropyrano[2,3-c]pyrazole (5a)}

Determination of the molecular structure of the organic molecules provides insight into the important molecular properties. Knowledge of the electronic structure and the spectral properties help in understanding the biological activity. Density functional theory (DFT) is a quantum mechanical theory used 
Table 4

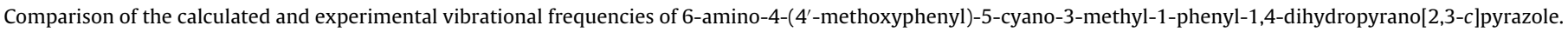

\begin{tabular}{|c|c|c|c|c|c|c|c|}
\hline \multirow[t]{2}{*}{ Mode } & \multicolumn{3}{|l|}{ Calculated } & \multicolumn{2}{|l|}{ Experimental } & \multirow[t]{2}{*}{$\%$ PED } & \multirow[t]{2}{*}{ Approximate character } \\
\hline & Freq $\left(\mathrm{cm}^{-1}\right)$ & $\begin{array}{l}\text { IR intensity } \\
\left(\mathrm{cm}^{-1}\right)\end{array}$ & $\begin{array}{l}\text { Raman } \\
\text { intensity } \\
\left(\mathrm{cm}^{-1}\right)\end{array}$ & IR freq $\left(\mathrm{cm}^{-1}\right)$ & $\begin{array}{l}\text { Raman freq } \\
\left(\mathrm{cm}^{-1}\right)\end{array}$ & & \\
\hline 11 & 521 & 6.60 & 0.69 & 523 & 511 & $\begin{array}{l}\tau \text { N28C19C7C6-25, } \tau \\
011 C 1 C 34 C 5-17\end{array}$ & Torsion of nitrile group \\
\hline 13 & 552 & 3.09 & 2.24 & 565 & 567 & $\begin{array}{l}\delta \mathrm{C} 1 \mathrm{C} 5 \mathrm{C} 34-17, \delta \\
\mathrm{C} 12 \mathrm{O} 11 \mathrm{C} 5-10, \tau \\
\mathrm{N} 28 \mathrm{C} 19 \mathrm{C} 7 \mathrm{C} 6-12\end{array}$ & Phenyl ring def \\
\hline 14 & 618 & 2.09 & 7.81 & 614 & 613 & $\begin{array}{l}\delta \mathrm{C} 4 \mathrm{C} 34 \mathrm{C} 5-18, \delta \\
\mathrm{C} 2 \mathrm{C} 1 \mathrm{C} 5-24\end{array}$ & $\begin{array}{l}\text { In plane bending } \\
\text { phenyl ring }\end{array}$ \\
\hline 15 & 631 & 7.69 & 0.97 & 630 & 634 & $\begin{array}{l}\delta \mathrm{N} 28 \mathrm{C} 19 \mathrm{C} 7-10, \delta \\
\mathrm{C} 19 \mathrm{C} 7 \mathrm{C} 9-20\end{array}$ & $\begin{array}{l}\text { NCC bending of nitrile } \\
\text { group }\end{array}$ \\
\hline 19 & 671 & 1.40 & 0.50 & 668 & 678 & $\begin{array}{l}\tau \text { N17N16C10C8-18, } \tau \\
\text { H26C24C18C8-11, } \tau \\
\text { C18N17N16C10-16 }\end{array}$ & Pyrazole ring torsion \\
\hline 20 & 697 & 1.13 & 1.03 & 721 & 706 & $\begin{array}{l}\tau \mathrm{C} 4 \mathrm{C} 34 \mathrm{C} 5 \mathrm{C} 1-10, \tau \\
\mathrm{C} 2 \mathrm{C} 1 \mathrm{C} 5 \mathrm{C} 34-10, \tau \\
\mathrm{C} 3 \mathrm{C} 2 \mathrm{C} 1 \mathrm{C} 5-24\end{array}$ & Phenyl ring torsion \\
\hline 22 & 760 & 5.33 & 3.05 & 750 & 748 & $\begin{array}{l}\nu \mathrm{C} 1 \mathrm{C} 5-11 \\
v \mathrm{O} 11 \mathrm{C} 5-15 \\
\delta \mathrm{C} 3 \mathrm{C} 2 \mathrm{C} 1-10\end{array}$ & $\begin{array}{l}\text { OC stretch ether } \\
\text { Linkage }+ \text { CC } \\
\text { Stretching }\end{array}$ \\
\hline 30 & 966 & 22.6 & 2.86 & 971 & 936 & $\begin{array}{l}\nu \mathrm{N} 17 \mathrm{C} 18-10, \delta \\
\mathrm{C} 18 \mathrm{~N} 17 \mathrm{~N} 16-14, \delta \\
\mathrm{H} 25 \mathrm{C} 24 \mathrm{H} 27-11, \tau \\
\mathrm{N} 25 \mathrm{C} 24 \mathrm{C} 18 \mathrm{C} 8-21, \tau \\
\mathrm{H} 27 \mathrm{C} 24 \mathrm{C} 18 \mathrm{C} 8-20\end{array}$ & $\begin{array}{l}\text { Methyl ring wagging } \\
\text { attached to pyrazole } \\
\text { ring }\end{array}$ \\
\hline 32 & 1006 & 49.27 & 0.97 & 1001 & 1003 & $\begin{array}{l}\nu \text { O29C9-32, } \delta \\
\mathrm{H} 22 \mathrm{~N} 20 \mathrm{C} 9-23\end{array}$ & $\begin{array}{l}\text { O-C stretch of pyran } \\
\text { ring }\end{array}$ \\
\hline 34 & 1035 & 48.8 & 2.49 & 1029 & 1.57 & v 011C12-72 & $\begin{array}{l}\mathrm{O}-\mathrm{C} \text { stretch of ether } \\
\text { attached to phenyl ring }\end{array}$ \\
\hline 36 & 1092 & 3.10 & 2.87 & 1073 & 1083 & $v \mathrm{~N} 17 \mathrm{~N} 16-41$ & $\begin{array}{l}\text { NN stretch of pyrazole } \\
\text { ring }\end{array}$ \\
\hline 42 & 1164 & 7.29 & 5.03 & 1169 & 1169 & $\delta \mathrm{H} 14 \mathrm{C} 12 \mathrm{H} 13-15$ & $\begin{array}{l}\text { Out of plane } \\
\text { bending + torsion of } \\
\text { methyl group attached } \\
\text { to ether linkage }\end{array}$ \\
\hline & & & & & & $\begin{array}{l}\tau \mathrm{H} 13 \mathrm{C} 12 \mathrm{O} 11 \mathrm{C} 5-28, \tau \\
\mathrm{H} 14 \mathrm{C} 12 \mathrm{O} 11 \mathrm{C} 5-29\end{array}$ & \\
\hline 45 & 1235 & 1.36 & 7.14 & 1223 & 1222 & $\begin{array}{l}\nu \mathrm{C} 3 \mathrm{C} 2-15, \delta \\
\mathrm{H} 33 \mathrm{C} 6 \mathrm{C} 3-31\end{array}$ & $\begin{array}{l}\text { CC stretching in phenyl } \\
\text { ring + HCC asym } \\
\text { bending }\end{array}$ \\
\hline 49 & 1291 & 19.3 & 1.69 & 1301 & 1313 & $\begin{array}{l}\delta \mathrm{H} 30 \mathrm{C} 1 \mathrm{C} 2-13, \delta \\
\mathrm{H} 31 \mathrm{C} 2 \mathrm{C} 3-14, \delta \\
\mathrm{H} 32 \mathrm{C} 4 \mathrm{C} 34-14, \delta \\
\mathrm{H} 35 \mathrm{C} 34 \mathrm{C} 5-11\end{array}$ & Phenyl ring bending \\
\hline 51 & 1362 & 2.2 & 8.16 & $1328 v w$ & $1363 \mathrm{vw}$ & $\begin{array}{l}\mathrm{H} 27 \mathrm{C} 24 \mathrm{H} 26-25, \\
\mathrm{H} 26 \mathrm{C} 24 \mathrm{H} 25-22, \\
\mathrm{H} 25 \mathrm{C} 24 \mathrm{H} 27-23\end{array}$ & $\begin{array}{l}\mathrm{HCH} \text { in plane bending } \\
\text { methyl group attached } \\
\text { to pyrazole }\end{array}$ \\
\hline 53 & 1384 & 60.59 & 37.58 & 1392 & 1390 & $\begin{array}{l}\nu \mathrm{N} 17 \mathrm{C} 18-26, \delta \\
\mathrm{C} 18 \mathrm{~N} 17 \mathrm{~N} 16-11, \delta \\
\mathrm{H} 23 \mathrm{~N} 16 \mathrm{~N} 17-18\end{array}$ & $\begin{array}{l}\text { NC stretch }+ \text { CNN bend } \\
\text { in pyrazole ring (def) }\end{array}$ \\
\hline 56 & 1436 & 5.54 & 19.98 & 1442 & 1392 & $\delta \mathrm{H} 27 \mathrm{C} 24 \mathrm{H} 26-44$ & $\begin{array}{l}\text { HCC sym bending } \\
\text { methyl group attached } \\
\text { to pyrazole ring }\end{array}$ \\
\hline & & & & & & $\begin{array}{l}\delta \mathrm{H} 26 \mathrm{C} 24 \mathrm{H} 25-31 \\
\tau \mathrm{H} 26 \mathrm{C} 24 \mathrm{C} 18 \mathrm{C} 8-1\end{array}$ & \\
\hline 57 & 1440 & 5.9 & 20.37 & 1443 & 1441 & $\begin{array}{l}\delta \mathrm{H} 25 \mathrm{C} 24 \mathrm{H} 27--37, \delta \\
\mathrm{H} 26 \mathrm{C} 24 \mathrm{H} 25-15\end{array}$ & $\begin{array}{l}\mathrm{HCC} \text { asym bending } \mathrm{CH}_{3} \\
\text { group attached to } \\
\text { pyrazole ring }\end{array}$ \\
\hline 59 & 1460 & 31.29 & 7.15 & 1465 & 1440 & $\delta \mathrm{H} 14 \mathrm{C} 12 \mathrm{H} 13-39$ & $\begin{array}{l}\text { Methyl group bending } \\
\text { (methoxy group) }\end{array}$ \\
\hline 62 & 1513 & 173.13 & 12.52 & 1512 & 1549 & $\begin{array}{l}\nu \mathrm{N} 16 \mathrm{C} 10-31, v \\
\mathrm{O} 29 \mathrm{C} 10-17, \delta \\
\mathrm{H} 23 \mathrm{~N} 16 \mathrm{~N} 17-15, \delta \\
\mathrm{N} 16 \mathrm{C} 10 \mathrm{C} 8-14\end{array}$ & $\begin{array}{l}\text { NC stretch }+\mathrm{HNN} \\
\text { bending in pyrazole } \\
\text { ring }+\mathrm{OC} \text { stretching } \\
\text { pyran ring }\end{array}$ \\
\hline 65 & 1595 & 9.63 & 4.64 & 1582 & 1542 & $\begin{array}{l}\nu \mathrm{C} 10 \mathrm{C} 8-23, \delta \\
\mathrm{H} 21 \mathrm{~N} 20 \mathrm{H} 22-24\end{array}$ & $\begin{array}{l}\mathrm{CC} \text { stretch of pyran } \\
\text { ring + out of plane } \mathrm{NH}_{2} \\
\text { bend }\end{array}$ \\
\hline 66 & 1605 & 86.17 & 82.16 & 1597 & 1584 & $\begin{array}{l}\text { v C } 4 \text { C } 34-30 \\
v \text { C } 2 \text { C } 1-11 \\
v \text { C } 3 C 2-11\end{array}$ & Phenyl ring breathing \\
\hline 67 & 1640 & 444.70 & 55.44 & 1641 & 1628 & $\begin{array}{l}\nu \mathrm{C} 7 \mathrm{C} 9-36, v \\
\mathrm{C} 10 \mathrm{C} 8-11, \\
\nu \mathrm{N} 20 \mathrm{C} 9-11, \delta \\
\mathrm{H} 21 \mathrm{~N} 20 \mathrm{H} 22-13\end{array}$ & $\begin{array}{l}\text { Ring breathing of pyran } \\
\text { ring }+\mathrm{NH}_{2} \text { bend }\end{array}$ \\
\hline
\end{tabular}


Table 4 (Continued)

\begin{tabular}{|c|c|c|c|c|c|c|c|}
\hline \multirow[t]{2}{*}{ Mode } & \multicolumn{3}{|l|}{ Calculated } & \multicolumn{2}{|l|}{ Experimental } & \multirow[t]{2}{*}{$\%$ PED } & \multirow[t]{2}{*}{ Approximate character } \\
\hline & Freq $\left(\mathrm{cm}^{-1}\right)$ & $\begin{array}{l}\text { IR intensity } \\
\left(\mathrm{cm}^{-1}\right)\end{array}$ & $\begin{array}{l}\text { Raman } \\
\text { intensity } \\
\left(\mathrm{cm}^{-1}\right)\end{array}$ & IR freq $\left(\mathrm{cm}^{-1}\right)$ & $\begin{array}{l}\text { Raman freq } \\
\left(\mathrm{cm}^{-1}\right)\end{array}$ & & \\
\hline 68 & 2222 & 66.95 & 192.72 & 2191vs & 2191vs & $\begin{array}{l}\nu \mathrm{N} 28 \mathrm{C} 19-89, v \mathrm{C} 19 \\
\mathrm{C} 7-11\end{array}$ & $\begin{array}{l}\mathrm{CN} \text { sym stretch of } \\
\text { nitrile group }\end{array}$ \\
\hline 69 & 2884 & 22.49 & 65.68 & $2856 \mathrm{~m}$ & $2839 m$ & $v \mathrm{C} 6 \mathrm{H} 33100$ & $\mathrm{CH}$ stretch \\
\hline 70 & 2898 & 56.53 & 123.49 & 2836 & 2882 & $\begin{array}{l}v \mathrm{C} 12 \mathrm{H} 13-45, v \\
\mathrm{C} 12 \mathrm{H} 14-47\end{array}$ & $\begin{array}{l}\mathrm{CH} \text { sym stretch of } \\
\text { methyl group attached } \\
\text { to ether linkage }\end{array}$ \\
\hline 71 & 2929 & 17.17 & 122.53 & 2925 & 2937 & $\begin{array}{l}v \mathrm{C} 24 \mathrm{H} 25, v \mathrm{C} 24 \\
\mathrm{H} 26-17, v \mathrm{C} 24 \mathrm{H} 27-30\end{array}$ & $\begin{array}{l}\mathrm{CH} \text { sym stretch of } \\
\text { methyl group attached } \\
\text { pyrazole ring }\end{array}$ \\
\hline 72 & 2957 & 40.72 & 48.80 & 2962 & 2935 & $\begin{array}{l}v \mathrm{C} 12 \mathrm{H} 13-51, v \\
\mathrm{C} 12 \mathrm{H} 14-49\end{array}$ & $\begin{array}{l}\mathrm{CH} \text { asym stretch of } \\
\text { methyl group attached } \\
\text { to ether linkage. }\end{array}$ \\
\hline 76 & 3054 & 11.02 & 59.77 & 3053 & 3050 & $v \mathrm{C} 4 \mathrm{H} 3295$ & $\begin{array}{l}\mathrm{CH} \text { asym stretch } \\
\text { phenyl ring }\end{array}$ \\
\hline 79 & 3097 & 11.28 & 88.90 & 3104 & 3078 & $v \mathrm{C} 1-\mathrm{H} 3095$ & $\begin{array}{l}\mathrm{CH} \text { sym stretch phenyl } \\
\text { ring }\end{array}$ \\
\hline 80 & 3445 & 63.14 & 175.11 & 3481 & 3482 & $\begin{array}{l}v \mathrm{~N} 20 \mathrm{H} 21-57, v \\
\mathrm{~N} 20-\mathrm{H} 22-43\end{array}$ & $\begin{array}{l}\mathrm{NH} \text { sym stretch of } \mathrm{NH}_{2} \\
\text { group attached to } \\
\text { pyran ring }\end{array}$ \\
\hline 81 & 3545 & 119.74 & 165.22 & 3565 & & $v \mathrm{~N} 16-\mathrm{H} 23-100$ & $\begin{array}{l}\mathrm{NH} \text { stretch of pyrazole } \\
\text { ring }\end{array}$ \\
\hline 82 & 3562 & 42.35 & 78.67 & 3587 & & $\begin{array}{l}v \mathrm{~N} 20 \mathrm{H} 2143, \\
v \mathrm{~N} 20-\mathrm{H} 2257\end{array}$ & $\begin{array}{l}\mathrm{NH} \text { asym stretch of } \\
\mathrm{NH}_{2} \text { group attached to } \\
\text { pyran ring }\end{array}$ \\
\hline
\end{tabular}

$\delta$, bend; $\tau$, torsion; $v$, stretch; asym, asymmetric; sym, symmetric; def, deformation.

to investigate the electronic structure in the ground state of many-body systems, in particular atoms and molecules. Raman spectroscopy has recently been proved to be a valuable tool in the investigation of complex molecules of biological interest [15-17].

Recently, vibrational spectral studies and theoretical computations related to natural coumarin derivatives $[18,19]$, ginkgolide [20], cresyl violet perchlorate [21], and molecules like 2,4-dichloro6-nitrophenol [22] have appeared in the literature. Atalay et al. $[23,24]$ have reported the theoretical studies on molecular structure and vibrational spectra of 2-amino-5-phenyl-1,3,4-thiadiazole and 1-amino-5-benzoyl-4-phenylpyrimidin-2(1H). Yakuphanoglu et al. [25] and Sekerci et al. [26] have successfully used Hartree-Fock and density functional methods to study the spectral properties of molecules like $N$-phenyl- $N^{\prime}$-(2-thienylmethylene) hydrazine and 1-(thiophen-2-yl-methyl)-2-(thiophen-2-yl)-1Hbenzimidazole.
In this paper, we report the interpretation of vibrational spectra and computations related to the structure of 6-amino-4-(4'-methoxyphenyl)-5-cyano-3-methyl-1-phenyl-1,4dihydropyrano[2,3-c]pyrazole (5a, Fig. 1) using density functional theory.

\subsection{Computational details}

Density functional theory calculations were carried out using Gaussian 09 package [27]. Equilibrium structure and vibrational frequencies were optimized using Becke-3-Lee-Yang-Parr (B3LYP) functional with $6-31 \mathrm{~g}^{* *}$ basis set $[28,29]$. Frequency calculations were carried out on the optimized structure using the program available in the Gaussian software itself. Equilibrium symmetry predicted is C1 with energy of -1392.97 Hartrees. Potential energy distribution was carried out using VEDA 4.0 program [30]. Opti-

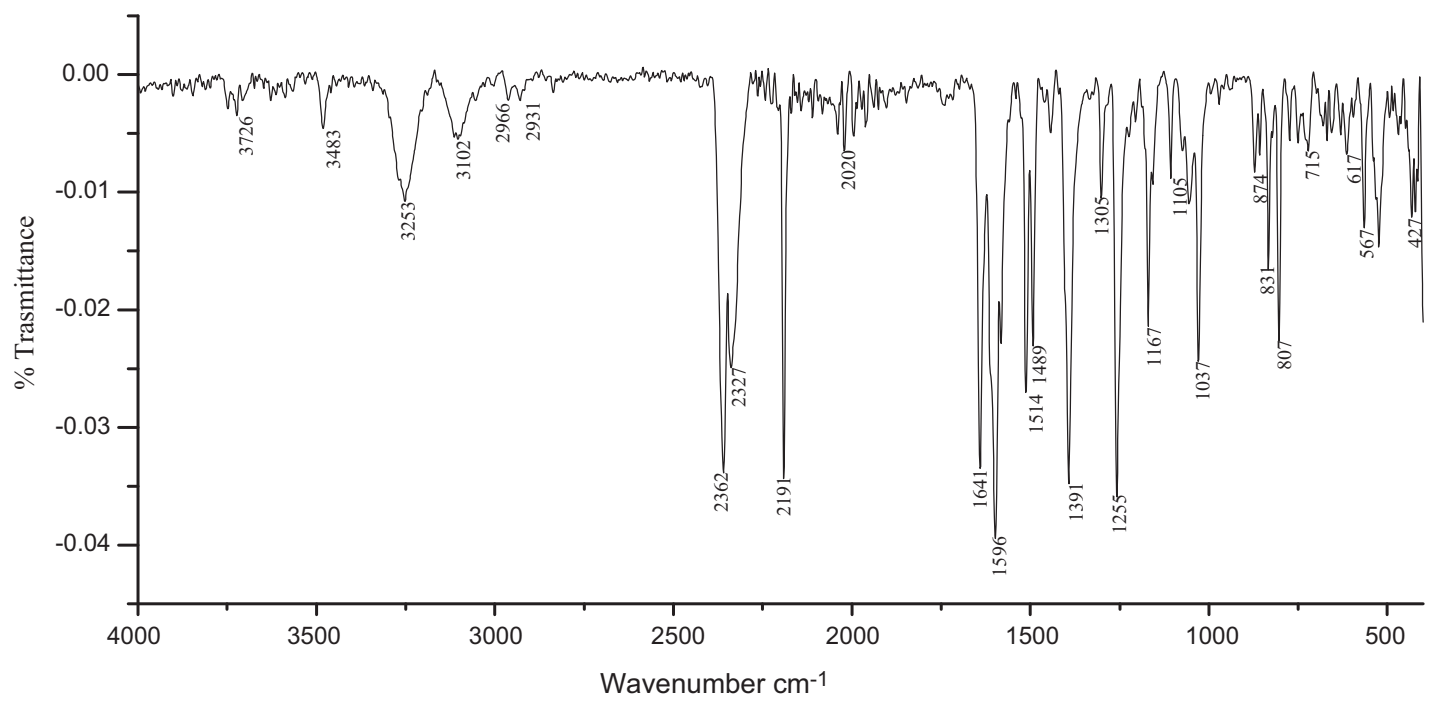

Fig. 3. FT-IR spectrum of 6-amino-4-(4'-methoxyphenyl)-5-cyano-3-methyl-1-phenyl-1,4-dihydropyrano[2,3-c]pyrazole in 400-4000 $\mathrm{cm}^{-1}$. 


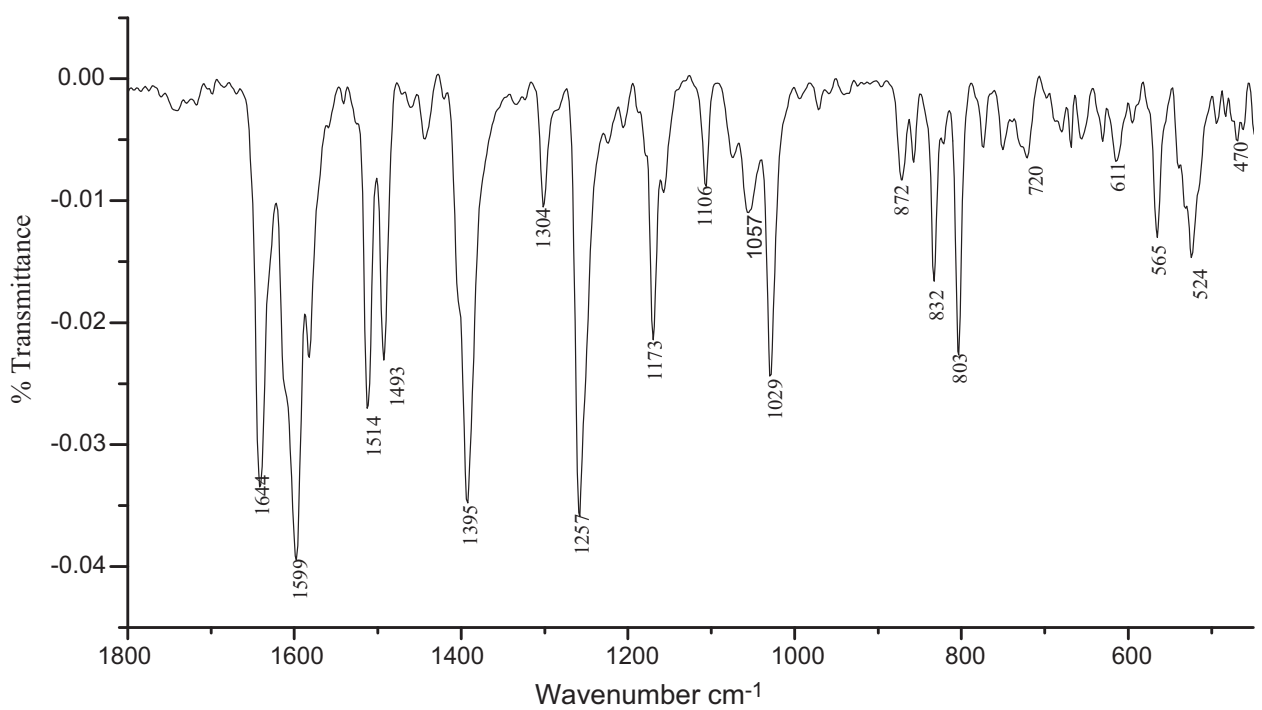

Fig. 4. FT-IR spectrum of 6-amino-4-(4'-methoxyphenyl)-5-cyano-3-methyl-1-phenyl-1,4-dihydropyrano[2,3-c]pyrazole in $1800-400 \mathrm{~cm}^{-1}$.

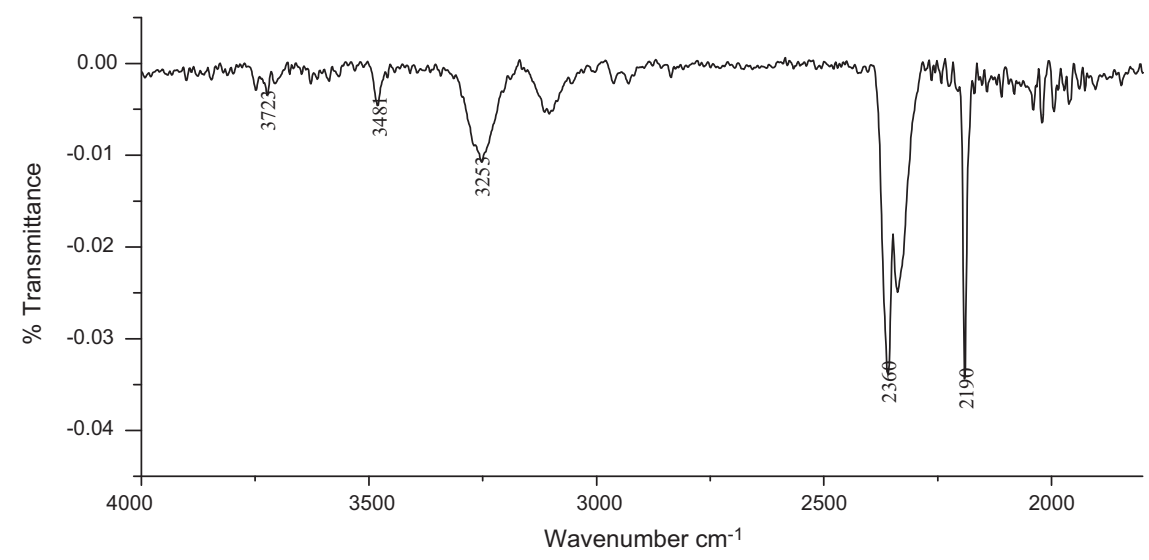

Fig. 5. FT-IR spectrum of 6-amino-4-(4'-methoxyphenyl)-5-cyano-3-methyl-1-phenyl-1,4-dihydropyrano[2,3-c]pyrazole in 4000-1800 $\mathrm{cm}^{-1}$.

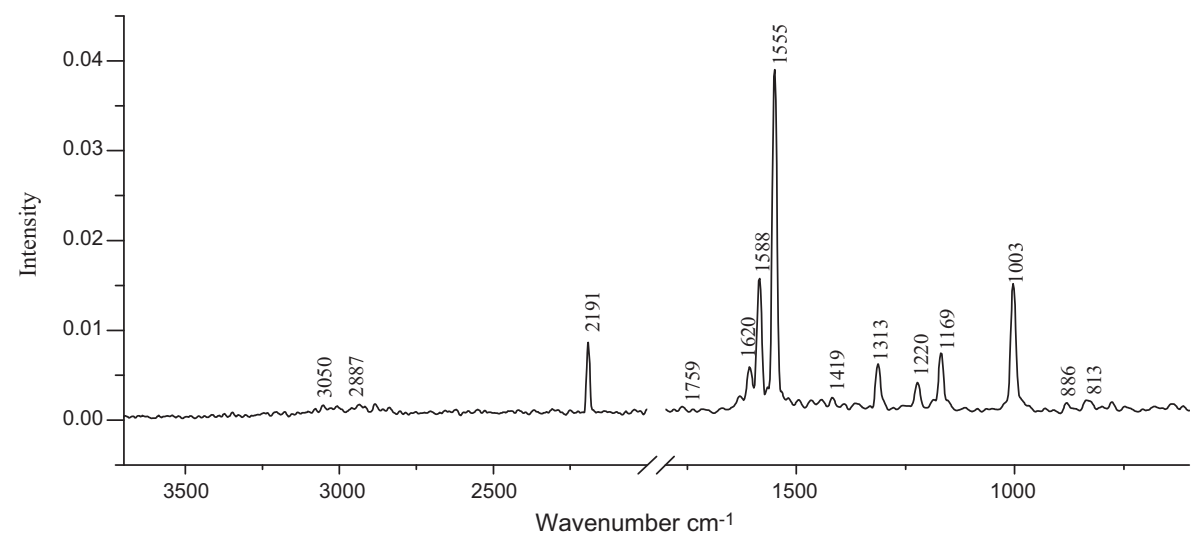

Fig. 6. FT-Raman spectrum of 6-amino-4-(4'-methoxyphenyl)-5-cyano-3-methyl-1-phenyl-1,4-dihydropyrano[2,3-c]pyrazole in 3700-600 $\mathrm{cm}^{-1}$.

mized geometry of the molecule and the numbering system is given in Fig. 2, optimized bond lengths and bond angles are presented in Table 2 and optimized dihedral angles are given in Table 3.

\section{Vibrational analysis}

6-Amino-4-(4'-methoxyphenyl)-5-cyano-3-methyl-1-phenyl1,4-dihydropyrano[2,3-c]pyrazole has 35 atoms, hence, 99 modes of fundamental vibrations are possibe. Predicted equilibrium symmetry for this molecule is C1 with energy of -1392.97 hartrees. A detailed vibrational analysis has been carried out and assignments of the observed fundamental bands have been proposed on the basis of peak positions and the relative intensities. The PED contribution to each of the observed frequencies gives the reliability and precision of the spectral analysis. The calculated harmonic-vibrational frequencies and the observed FT-IR and FT- 


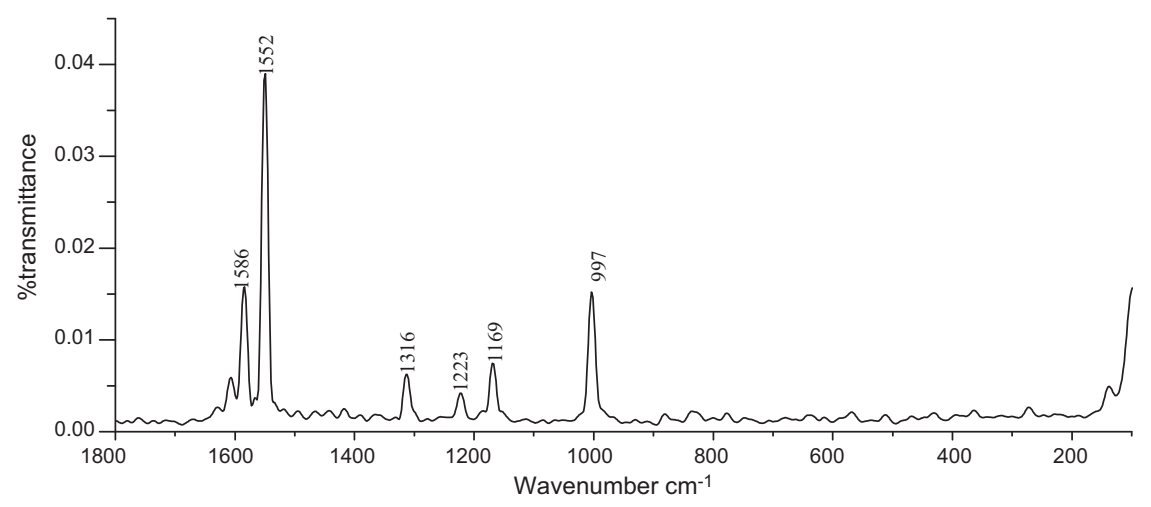

Fig. 7. FT-Raman spectrum of 6-amino-4-(4'-methoxyphenyl)-5-cyano-3-methyl-1-phenyl-1,4-dihydropyrano[2,3-c]pyrazole in 1800-100 cm-1 .

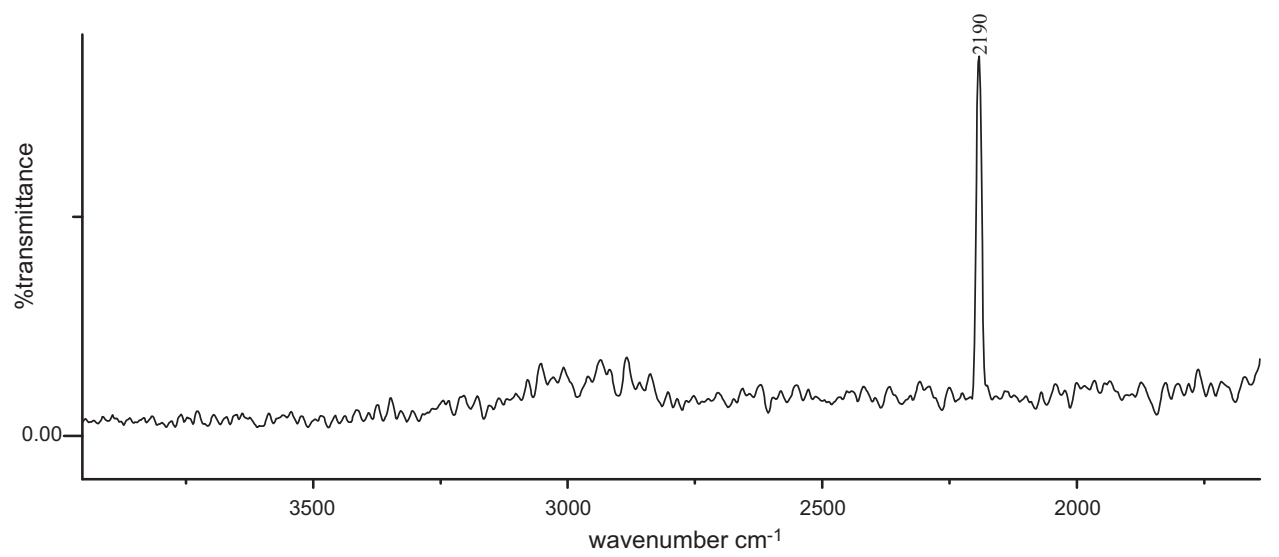

Fig. 8. FT-Raman spectrum of 6-amino-4-(4'-methoxyphenyl)-5-cyano-3-methyl-1-phenyl-1,4-dihydropyrano[2,3-c]pyrazole in 3700-1800 cm-1 .

Raman frequencies for various modes of vibrations are presented in Table 4. A comparison of the calculated frequencies with the experimental values indicates the overestimation of the calculated vibrational modes due to neglect of anharmonicity in real system. Hence, computed frequencies were multiplied with scale factor 0.9614 to offset the systematic error caused by neglecting anharmonicity giving a good agreement with the observed frequencies. These calculations provide a valuable insight into the vibrational spectrum and molecular parameters.

6-Amino-4-(4'-methoxyphenyl)-5-cyano-3-methyl-1phenyl-1,4-dihydropyrano[2,3-c]pyrazole (5a) has a pyrazole ring with a methyl substituent, pyran ring has a nitrile, an amino group and a phenyl ring with a p-methoxy substituent. The pyran (C10-C8-C6-C7-C9-O29) and the pyrazole(C10-C8-C18-N16-N17) are fused together and lie in one plane. The phenyl ring $(\mathrm{C} 1-\mathrm{C} 2-\mathrm{C} 3-\mathrm{C} 4-\mathrm{C} 34-\mathrm{C} 5)$ lies perpendicular to the plane of the fused rings ( $\mathrm{LC} 3-\mathrm{C} 6-\mathrm{C} 7=112^{\circ}$, Fig. 2 ). Hence, the vibrational spectral analysis was performed based on the characteristic vibrations of the methyl group, amino group, nitrile group, methoxy group, and a phenyl ring. The FT-IR and FT-Raman spectra are shown in Figs. 3-8.

\subsection{Methyl group vibrations}

The molecule under consideration has two methyl groups, one attached to the pyrazole ring and the other to the side chain phenyl ring as methoxy substituent. The asymmetric stretching mode of methyl group is expected to be around $2980 \mathrm{~cm}^{-1}$ and symmetric stretching at $2870 \mathrm{~cm}^{-1}[31,32]$. In our molecule, C-H symmetric stretch of methyl group vibrations recorded at $2836 \mathrm{~cm}^{-1}$, $2925 \mathrm{~cm}^{-1}$ (FT-IR) and $2882 \mathrm{~cm}^{-1}, 2937 \mathrm{~cm}^{-1}$ (FT-Raman) are consistent with the predicted values of $2898 \mathrm{~cm}^{-1}, 2929 \mathrm{~cm}^{-1}$. $\mathrm{C}-\mathrm{H}$ asymmetric stretching vibrations computed at $2957 \mathrm{~cm}^{-1}$ and $2984 \mathrm{~cm}^{-1}$ match with the $2963 \mathrm{~cm}^{-1}, 3005 \mathrm{~cm}^{-1}$ in IR and $2957 \mathrm{~cm}^{-1}, 3007 \mathrm{~cm}^{-1}$ in Raman spectrum.

The symmetric and asymmetric bending vibrations of methyl group are normally expected in the regions $1465-1440 \mathrm{~cm}^{-1}$ and $1390-1370 \mathrm{~cm}^{-1}$, respectively. The asymmetric bending of methyl group observed at $1465 \mathrm{~cm}^{-1}$ (IR) and $1440 \mathrm{~cm}^{-1}$ (Raman) are in good agreement with the theoretically calculated value of $1460 \mathrm{~cm}^{-1}$. The symmetric bending calculated at $1436 \mathrm{~cm}^{-1}$ is observed as a medium band at $1442 \mathrm{~cm}^{-1}$ (IR) and $1392 \mathrm{~cm}^{-1}$ (Raman). Out of plane bending with torsion of methyl group attached to ether linkage is calculated at $1164 \mathrm{~cm}^{-1}$ and is experimentally seen at $1169 \mathrm{~cm}^{-1}$ in IR and Raman.

\section{2. $\mathrm{NH}_{2}$ and $\mathrm{NH}$ group vibrations}

The $\mathrm{NH}_{2}$ wagging was computed at $496 \mathrm{~cm}^{-1}$ and is observed at $519 \mathrm{~cm}^{-1}$ in FT-IR and at $512 \mathrm{~cm}^{-1}$ in FT-Raman. NH sym stretch of $\mathrm{NH}_{2}$ group attached to pyran ring is calculated at $3445 \mathrm{~cm}^{-1}$ is in good agreement with the experimental values of $3481 \mathrm{~cm}^{-1}$ and $3482 \mathrm{~cm}^{-1}$ in FT-IR and FT-Raman, respectively. NH asym stretch of $\mathrm{NH}_{2}$ group attached to pyran ring which is calculated to appear at $3562 \mathrm{~cm}^{-1}$ is observed only in FT-IR at $3581 \mathrm{~cm}^{-1}$ and not in FTRaman. NH stretch of pyrazole ring is calculated as $3545 \mathrm{~cm}^{-1}$ is also seen only in FT-IR at $3565 \mathrm{~cm}^{-1}$.

\section{3. $C=N, C-N, N-N$ and the nitrile group}

$\mathrm{C}=\mathrm{N}$ and $\mathrm{C}-\mathrm{N}$ stretching vibrations generally occur in the region $1382-1266 \mathrm{~cm}^{-1}$, and it is difficult to assign these bands as they 
get mixed with several other bands. In our study, the pyrazole ring contains the $\mathrm{C}=\mathrm{N}$ and $\mathrm{C}-\mathrm{N}$ bonds, which show stretching vibrations at $1392,1512 \mathrm{~cm}^{-1}$ in IR and at $1390,1549 \mathrm{~cm}^{-1}$ in Raman. These are consistent with the calculated frequencies at $1384 \mathrm{~cm}^{-1}$ and $1513 \mathrm{~cm}^{-1}$ along with the bending vibrations.

A characteristic band for the nitrile group attached to the pyran ring is observed at $2191 \mathrm{~cm}^{-1}$ in both FT-IR and FT-Raman spectra, which is in very good agreement with the computed value of $2222 \mathrm{~cm}^{-1}$. Torsion of nitrile group calculated at $521 \mathrm{~cm}^{-1}$ agrees with the observed values of $523 \mathrm{~cm}^{-1}$ in IR and $511 \mathrm{~cm}^{-1}$ in Raman.

$\mathrm{N}-\mathrm{N}$ Stretch of pyrazole ring was calculated at $1092 \mathrm{~cm}^{-1}$ and is consistent with the experimental values of $1073 \mathrm{~cm}^{-1}$ in IR and $1083 \mathrm{~cm}^{-1}$ in Raman spectrum.

\section{4. $\mathrm{C}-\mathrm{H}$ vibrations}

Generally, C-H stretching, $\mathrm{C}-\mathrm{H}$ in-plane bending and $\mathrm{C}-\mathrm{H}$ outof-plane bending vibrations appear in the range $3100-3000 \mathrm{~cm}^{-1}$, $1300-1000 \mathrm{~cm}^{-1}$ and $1000-750 \mathrm{~cm}^{-1}$ [33]. HCH in plane bending of methyl group attached to pyrazole ring is calculated at $1362 \mathrm{~cm}^{-1}$ is experimentally observed at $1328 \mathrm{~cm}^{-1}$ (IR) and $1363 \mathrm{~cm}^{-1}$ (Raman) as very weak bands. $\mathrm{CH}$ stretch of hydrogen attached to pyran ring is observed at $2856 \mathrm{~cm}^{-1}$ in IR and at $2839 \mathrm{~cm}^{-1}$ in Raman spectrum matches the predicted frequency of $2884 \mathrm{~cm}^{-1}$.

\subsection{C-O group vibrations}

$\mathrm{C}_{15} \mathrm{H}_{14} \mathrm{~N}_{4} \mathrm{O}_{2}$ has two ether linkages, one in the form of cyclic ether in pyran ring and the other one as methoxy group attached to the phenyl ring. $\mathrm{O}-\mathrm{C}$ stretch of these two are calculated at $1035 \mathrm{~cm}^{-1}\left(\mathrm{O}-\mathrm{CH}_{3}\right)$ and $1044 \mathrm{~cm}^{-1}$ (cyclic ether). These have been found to be consistent with the recorded values at $1029 \mathrm{~cm}^{-1}$, $1057 \mathrm{~cm}^{-1}$ (IR) and $1038 \mathrm{~cm}^{-1}$ (Raman). The calculated O-C Stretch of pyran ring $\left(\mathrm{O}-\mathrm{C}-\mathrm{NH}_{2}\right)$ at $1006 \mathrm{~cm}^{-1}$ coincides very well with the observed values of $1001 \mathrm{~cm}^{-1}$ and $1003 \mathrm{~cm}^{-1}$ in the IR and Raman spectra.

\subsection{Phenyl ring vibrations}

$\mathrm{C}-\mathrm{H}$ asymmetric stretching of phenyl ring is calculated at $3054 \mathrm{~cm}^{-1}$ and experimental value is at $3053 \mathrm{~cm}^{-1}$ and $3050 \mathrm{~cm}^{-1}$ in IR and Raman respectively. $\mathrm{C}-\mathrm{H}$ symmetric stretching of phenyl ring which is calculated at $3097 \mathrm{~cm}^{-1}$ is in good agreement with the value $3104 \mathrm{~cm}^{-1}$ in IR and $3078 \mathrm{~cm}^{-1}$ in Raman spectrum. Phenyl ring breathing observed at $1641 \mathrm{~cm}^{-1}$ and $1628 \mathrm{~cm}^{-1}$ agreed with the computed value of $1605 \mathrm{~cm}^{-1}$. Phenyl ring deformation computed at $552 \mathrm{~cm}^{-1}$ agreed with the recorded values of $565 \mathrm{~cm}^{-1}$ and $567 \mathrm{~cm}^{-1}$ in IR and Raman. In-plane bending of phenyl ring is calculated at $618 \mathrm{~cm}^{-1}$ and was observed at $614 \mathrm{~cm}^{-1}$ in IR and $613 \mathrm{~cm}^{-1}$ in Raman. Computed phenyl ring torsion at $697 \mathrm{~cm}^{-1}$ is in agreement with the observed values of $721 \mathrm{~cm}^{-1}$ and $706 \mathrm{~cm}^{-1}$ in IR and Raman spectra.

\section{Conclusions}

A convenient and efficient protocol has been developed for the synthesis of pyranopyrazoles in high yields using catalytic amounts of imidazole as an organocatalyst, in water. We have demonstrated that, the synthesis is milder, fast and does not require any tedious work-up procedure. An effort has been made in the present study to optimize the structure of 6-amino-4-(4'-methoxyphenyl)-5cyano-3-methyl-1-phenyl-1,4-dihydropyrano[2,3-c]pyrazole (5a, $\mathrm{C}_{15} \mathrm{H}_{14} \mathrm{~N}_{4} \mathrm{O}_{2}$ ). The geometric parameters and vibrational frequencies of 5a were calculated using B3LYP method with $6-31 \mathrm{~g}^{* *}$ basis set. To fit the theoretical results, the experimental values were multiplied by 0.9614 . The gained multiplication factors were found to be in good agreement with the experimental values for both FTIR and FT-Raman. These results confirm the validity of potential energy distribution to each of the observed frequencies.

Organic molecules possessing similar functional groups have been synthesized and their structures have been studied using DFT calculations and vibrational spectral analyses [34,35]. It has been found by us that, the vibrational frequency values reported for the corresponding functional groups are consistent with the values which we have observed for the functional groups present in pyranopyrazoles.

\section{Acknowledgments}

Aisha Siddekha gratefully acknowledges, The Indian Academy of Sciences, Bangalore, Indian National Science Academy, New Delhi, The National Academy of Sciences, Allahabad, for the short term Fellowship and The Indian Institute of Science, Bangalore, for helping her in the FT-Raman and FT-IR spectral analysis during Summer Research Fellowship programme during May to July 2010. The authors would also like to acknowledge the Sophisticated Instruments Facility for recording the NMR spectra and Archives Department, Indian Institute of Science, Bangalore, for editing the manuscript.

\section{References}

[1] A. Feurer, J. Luithle, S. Wirtz, G. Koenig, J.Stasch, E. Stahl, R. Schreiber, F. Wunder, D. Lang, PCT Int. Aool. Wo 2004009589, Baye Healtheare Ag, Germany.

[2] M.N. Nasr, M.M. Gineinah, Arch. Pharm. Med. Chem. 335 (2002) 289-295.

[3] V.K. Ahluwalia, A. Dahiya, V. Garg, Indian J. Chem. 36B (1997) 88-90.

[4] M.R. Nadia, Y.K. Nahed A., A. Fahmyb, A.A. F. El-Sayeda, Der Pharma Chem. 2 (2010) 400-417.

[5] H. Junek, Aigner, Chem. Ber. 106 (1973) 914-921.

[6] (a) H. Wamhoff, E. Kroth, K. Strauch, Synthesis 11 (1993) 1129-1132; (b) G. Tacconi, G. Gatti, G. Desimoni, V. Messori, J. Prakt. Chem. 322 (1980) 831-834;

(c) L.G. Sharanina, V.P. Marshtupa, A. Sharanin Yu, Khim. Geterosikl. Soedin. 10 (1980) 1420-1424.

[7] Y.A. Sharanin, L.G. Sharanina, V.V. Puzanova, Zh. Org. Khim. 19 (1983) 2609-2615.

[8] G. Vasuki, K. Kandhasamy, Tetrahedron Lett. 49 (2008) 5636-5638.

[9] (a) E.S. El-Tamany, F.A. El-Shahed, B.H. Mohamed, J. Serb. Chem. Soc. 64 (1999) 9-18;

(b) F.M. Abdelrazek, P. Metz, N.H. Metwally, S.F. El-Mahrouky, Arch. Pharm. 339 (2006) 456-460.

[10] J. Zhu, H. Bienayme (Eds.), Multicomponent Reactions in the Total Synthesis of Natural Products, Wiley-VCH, Weinheim, 2005, pp. 342-397.

[11] (a) N.M. Evdokimov, A.S. Kireev, A.A. Yakovenko, M.Y. Antipin, I.V. Magedov, A. Kornienko, J. Org. Chem. 72 (2007) 3443-3453;

(b) H. Bienayme, C. Hulme, G. Oddon, P. Schmitt, Chem. Eur. J. 6 (2000) 3321-3329.

[12] Y.M. Litvinov, A.A. Shestopalov, L.A. Rodinovskaya, A.M. Shestopalov, J. Comb. Chem. 11 (2009) 914-919.

[13] A.M. Shestopalov, Y.M. Emeliyanova, A.A. Shestopalov, L.A. Rodinovskaya, Z.I. Niazimbetova, D.H. Evans, Tetrahedron 59 (2003) 7491-7496.

[14] (a) M.A. Pasha, V.P. Jayashankara, J. Pharmacol. Toxicol. 1 (6) (2006) 573-578; (b) M.A. Pasha, V.P. Jayashankara, Heterocycles (2006) 1017-1023;

(c) M.A. Pasha, V.P. Jayashankara, Heterocycl. Commun. 12 (2006) 61-66; (d) M.B. Madhusudana Reddy, A. Nizam, M.A. Pasha, Synth. Commun. 40 (2010) 3384-3389;

(e) M.B. Madhusudana Reddy, V.P. Jayashankara, M.A. Pasha, Synth. Commun. 40 (2010) 2930-2934.

[15] R.J.H. Clark, R.E. Hester (Eds.), Spectroscopy of Biological Systems, Advances in Spectroscopy, vol. 13, Wiley, Chichester, 1986.

[16] Proceedings of the XVI International Conference on Raman Spectroscopy, Wiley, Chichester, 1998.

[17] J.P. Abraham, I.H. Joe, V. George, O.F. Nielsen, V.S. Jayakumar, Spectrochim. Acta A Mol. Biomol. Spectrosc. 59 (2003) 193-199.

[18] I. Mihaylov, G. Georgieva, I. Bauer, I. Kostova, I. Manolov, N. Trendafilova, Int. J. Quant. Chem. 106 (2006) 1304-1315.

[19] I. Georgieva, N. Trendafilova, W. Kiefer, V.K. Rastogi, I. Kostova, Vib. Spectrosc. 44 (2007) 78-88.

[20] W.-L. Zhu, C.M. Puah, Z.-J. Tan, H.-L. Jiang, K.-X. Chen, R.-Y. Ji, J. Mol. Struct. (THEOCHEM) 528 (2000) 193-198.

[21] E. Vogel, A. Gbureck, W. Kiefer, J. Mol. Struct. 550-551 (2000) 177-190.

[22] N. Sundaraganesan, B. Anand, J.B. Dominic, Spectrochim. Acta A Mol. Biomol. Spectrosc. 65A (2006) 1053-1062. 
[23] Y. Atalay, F. Yakuphanoglu, M. Sekerci, D. Avcıa, A. Basoglu, Spectrochim. Acta A 64 (2006) 68-72.

[24] Y. Atalay, F. Yakuphanoglu, M. Sekerci, Spectrochim. Acta A 65 (2006) 964-968.

[25] F. Yakuphanoglu, Y. Atalay, M. Sekerci, Spectrochim. Acta A 66 (2007) 438-441.

[26] M. Sekerci, Y. Atalay, F. Yakuphanoglu, D. Avci, A. Basoglu, Spectrochim. Acta A 67 (2007) 503-508.

[27] M.J. Frisch, G.W. Trucks, H.B. Schlegel, G.E. Scuseria, M.A. Robb, J.R. Cheeseman, G. Scalmani, V. Barone, B. Mennucci, G.A. Petersson, H. Nakatsuji, M. Caricato, X. Li, H.P. Hratchian, A.F. Izmaylov, J. Bloino, G. Zheng, J.L. Sonnenberg, M. Hada, M. Ehara, K. Toyota, R. Fukuda, J. Hasegawa, M. Ishida, T. Nakajima, Y. Honda, O. Kitao, H. Nakai, T. Vreven, J.A. Montgomery Jr., J.E. Peralta, F. Ogliaro, M. Bearpark, J.J. Heyd, E. Brothers, K.N. Kudin, V.N. Staroverov, R. Kobayashi, J. Normand, K. Raghavachari, A. Rendell, J.C. Burant, S.S. Iyengar, J. Tomasi, M. Cossi, N. Rega, J.M. Millam, M. Klene, J.E. Knox, J.B. Cross, V. Bakken, C. Adamo, J. Jaramillo, R. Gomperts, R.E. Stratmann, O. Yazyev, A.J. Austin, R. Cammi, C. Pomelli, J.W Ochterski, R.L. Martin, K. Morokuma, V.G. Zakrzewski, G.A. Voth, P. Salvador,
J.J. Dannenberg, S. Dapprich, A.D. Daniels, O. Farkas, J.B. Foresman, J.V. Ortiz, J. Cioslowski, D.J. Fox, Gaussian 09, Revision A.02, Gaussian, Inc., Wallingford, CT, 2009.

[28] A.D. Becke, J. Chem. Phys. 98 (1993) 5648-5652.

[29] C. Lee, W. Yang, R.G. Parr, Phys. Rev. B 37 (1988) 785-789.

[30] M.H. Jamroz, Vibrational Energy Distribution Analysis, Warsaw, 2004, VEDA 4.0 PROGRAM

[31] B. Smith, Infrared Spectral Interpretation: A Systematic Approach, CRC Press, Washington, DC, 1998.

[32] J. Binoy, J.I. Hubert, V.S. Jayakumar, J. Raman Spectrosc. 36 (2005) 1091-1100.

[33] G. Varsanyi, Assignments of Vibrational Spectra of 700 Benzene Derivatives, Wiley, New York, 1974.

[34] J. Amamlanathan, I.H. Joe, I. Kostava, J. Raman Spectrosc. 41 (2010) 1076-1084

[35] J. Swaminathan, M. Ramalingam, V. Sethuraman, N. Sundaraganesan, S. Sebastian, M. Kurt, Spectrochim. Acta A 75 (2010) 183-190. 